\title{
Computer Simulation of Hydrophobic Hydration Forces on Stacked Plates at Short Range
}

\author{
A. Wallqvist ${ }^{\dagger}$ and B. J. Berne* \\ Department of Chemistry and Center for Biomolecular Simulation, Columbia University, \\ New York, New York 10027
}

Received: September 30, $1994^{\otimes}$

The potential of mean force between two large parallel hydrophobic oblate ellipsoidal plates in liquid water is determined by molecular dynamics. Each ellipsoid displaces approximately 40 water molecules and has major and minor axes of 3.1 and $9.3 \AA$, respectively, has a surface area of $650 \AA^{2}$, and interacts repulsively with the solvent water molecules. The potential of mean force is calculated from thermodynamic perturbation theory for a series of decreasing plate separations, using constant-pressure molecular dynamics. As the plates are moved together, they are first separated by three water layers and then by two, but for shorter distances, a dewetting transition occurs, and one water layer is never observed despite the fact that one can fit. As the plates are brought together, there is a corresponding weak oscillation in the potential of mean force corresponding to the removal of each water layer until the dewetting transition takes place, and for closer separations, the surrounding water molecules induce a constant average attractive force of $25(\mathrm{~kJ} / \mathrm{mol}) / \AA$ between the plates. This hydrophobic attraction is largely entropic in character, and the potential of mean force is found to be proportional to the area of the water-vacuum surface in this dewetting regime. The constant of proportionality is found to be smaller than the gas-liquid surface tension of the water model used. There is a very strong short-range driving force toward contact pairing.

\section{Introduction}

The usual view of the hydrophobic interaction is based on a simple picture. ${ }^{1-5}$ Because nonpolar molecules are relatively insoluble in water, it is usually assumed that there is a thermodynamic driving force in water that will drive two apolar spheres together to a much greater degree than would be the case if these two spheres were dissolved in a nonpolar solvent. This driving force has two possible sources. The entropy of solution of simple nonpolar solutes in water is negative (e.g., $\left.\Delta S_{\mathrm{Ar}}^{\circ}=-126 \mathrm{~J} /(\mathrm{mol} \mathrm{deg})\right)$. Most of this entropic change springs from the ordering of the water molecules in close proximity to the solute molecule. Since two solute molecules in contact will order fewer solvent molecules than two solventseparated solute molecules, the entropy change on bringing the solute molecules from a large separation into contact should be positive, and if enthalpy changes are unimportant, as they would be for hard-sphere solutes, this change in relative separation will lower the overall free energy of the solution. From this point of view, the driving force for hydrophobic aggregation is consistent with the solvent trying to minimize the exposed surface area of the solute molecules. If the attractive interaction between neighboring bulk water molecules is stronger than between the solute molecule and water, enthalpic contributions will increase this hydrophobic aggregation, and if it is weaker, enthalpic contributions will decrease it.

As persuasive as this picture of hydrophobicity is, it has long been known that computer simulations of inert gas molecules as well as simple integral equation theories of solvation in water give a different picture. As first surmised by Pratt and Chandler ${ }^{6.7}$ and as first shown by the computer simulations of Pangali et al. ${ }^{8,9}$ we now know that solvent-separated pairing of two "hard-sphere" inert gas particles in which the two spheres reside in adjoining clathrate cages is at least as probable as the contact pairing. This conclusion has been confirmed in later

+ Present address: NCI-FCRDC, Building 1052, Room 238, P.O. Box B, Frederick, MD 21702.

${ }^{\otimes}$ Abstract published in Advance ACS Abstracts, February 1, 1995. work. ${ }^{7,10-22}$ Similarly, an inert gas particle prefers to be separated from a flat wall by a layer of solvent. ${ }^{23,24}$ Thus, the simple picture alluded to above does not appear to give the whole truth. It should be noted that the simulations were performed using fixed charge models of water (like ST2, ${ }^{25}$ TIP4P, ${ }^{26}$ SPC, ${ }^{27}$ etc.).

Solute molecules much larger than a water molecule should induce a different hydration structure and orientation than small solute molecules. ${ }^{23,28-31}$ In this paper, we investigate the hydrophobic interaction between two large parallel oblate ellipsoids or platelike "molecules" by determining the potential of mean force between them as a function of their separation in a fixed charge model of water. Each plate is large enough to displace about 40 water molecules and to thus preclude the formation of two adjoining clathrate cages. This study provides a picture of hydration forces for distances closer than $15 \AA$. In an article in this issue, we report on the hydrophobic hydration of one of these plate molecules. ${ }^{32}$ The long-range nature of solvation forces found in experiments ${ }^{33-36}$ cannot be addressed in the present work due to limitations on system-size that can adequately be simulated.

We find that the solvent-induced part of the potential of mean force between the plates is strongly attractive at short separations and a weakly undulating function of separation at larger separations. Unlike the hydrophobic interaction of small apolar spheres, the water induces a very strong contact pairing between the large plates, probably because the water cannot support a clathrate structure. We find that the space between the molecules can be filled by two or more layers of water but never less than two layers. When the plates are moved close enough together that two water layers cannot fit between them, all of the interstitial water molecules are expelled. The distance at which this dewetting transition first occurs is called the dewetting distance. For separations smaller than the dewetting distance, the potential of mean force is found to be strongly attractive and to be directly proportional to the area of the vacuum-water interface formed by the void space between the 
plates with a constant of proportionality equal to $70 \%$ of the independently determined ${ }^{37}$ gas-water surface tension. The hydrophobic driving force is due to the system's need to minimize the surface area of the cavity between the plates.

All of the simulations are based on a recently devised potential model of the water-water interaction called the reduced effective representation (RER) ${ }^{40}$ This model bears a close family resemblance to central force models and exhibits the typical features of liquid water, i.e., a liquid composed of strong directional hydrogen bonds arranged in a loose tetrahedral framework. Since it is basically these ingredients that characterize liquid water, we believe that the results presented are robust with respect to the water model.

\section{Computational Procedures}

In this paper, we study the interactions between two large highly eccentric oblate ellipsoids and water. Since we are primarily interested in the solvent response to the hydrophobic ellipsoids, we have chosen to use a purely repulsive waterellipsoid interaction, modeled as the repulsive part of the Lennard-Jones interaction of the Gay-Berne potential, ${ }^{41,32}$

$$
U_{\mathrm{sw}}=k T\left(\frac{\sigma_{0}}{r-\sigma(\chi ; \nu)+\sigma_{0}}\right)^{12}
$$

$\sigma(\chi ; v)$ represents a modified Lennard-Jones $\sigma$ parameter that depends parametrically on the anisotropy, $\chi$, of the oblate ellipsoid and on $v$, the angle between a vector from the center of the hydrophobic object to a water oxygen atom, $\hat{\mathbf{r}}$, and the major axis of the oblate ellipse, $\mathbf{0}$,

$$
v=\arccos (\hat{\mathbf{r}} \cdot \hat{\mathbf{u}})
$$

The functional form of $\sigma(\chi ; \nu)$ is that of an ellipse interacting with a sphere, ${ }^{42}$

$$
\sigma(\chi ; \nu)=\frac{\sigma_{\perp}}{\left(1-\chi \cos ^{2} \nu\right)^{1 / 2}}
$$

where $\chi$ is given by

$$
\chi=\frac{\sigma_{\|}^{2}-\sigma_{\perp}^{2}}{\sigma_{\|}^{2}}
$$

For $\chi=0$, the ellipsoid becomes a sphere of solvent excluded radius $\sigma(0 ; v)=R$, and the model interactions then collapse into the standard repulsive Lennard-Jones interaction. The potential is described by the anisotropy of the ellipsoid and the radius of the spherical object, $R$, which determines the volume of the hydrophobic object. As an illustration, equipotential surfaces ( $U_{\mathrm{sw}}=k T$ ) are drawn in Figure 1. The volume of the ellipsoid is the same for all solute-solvent interactions.

Our two hydrophobic plates are modeled by parameters, $R$ $=6.45 \AA$ and $\chi=-8$, that correspond to oblate ellipsoids with $\sigma_{\perp}=9.3 \AA$ and $\sigma_{||}=3.1 \AA$. This mimics an extended shape that has the same thickness as one layer of methane molecules. Each half side of this ellipse has an approximate surface area of $325 \AA^{2}$, an area which would require $\approx 25$ water molecules to fully hydrate it.

In the simulation of the liquid water-solute system, 1656 water molecules were used in order to fully hydrate the two hydrophobic plates at their maximum separation. This allows for a maximum hydrated conformation where three water layers are accommodated between the plates. In Figure 2, a coordinate projection of such a configuration is given. The interaction between the solute molecules themselves need not be specified,

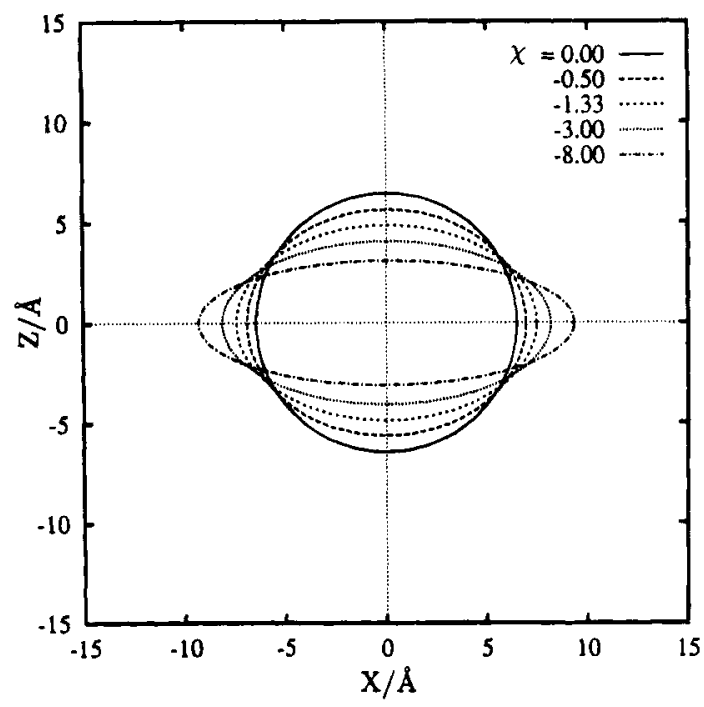

Figure 1. Cut through the equipotential surface, $U_{\mathrm{sw}}=k T$, in the plane of the minor and major axes, for various values of the eccentricity, $\chi$, given that $R=6.45 \AA$. The solid of revolution around the major axis remains the same during the contortion.

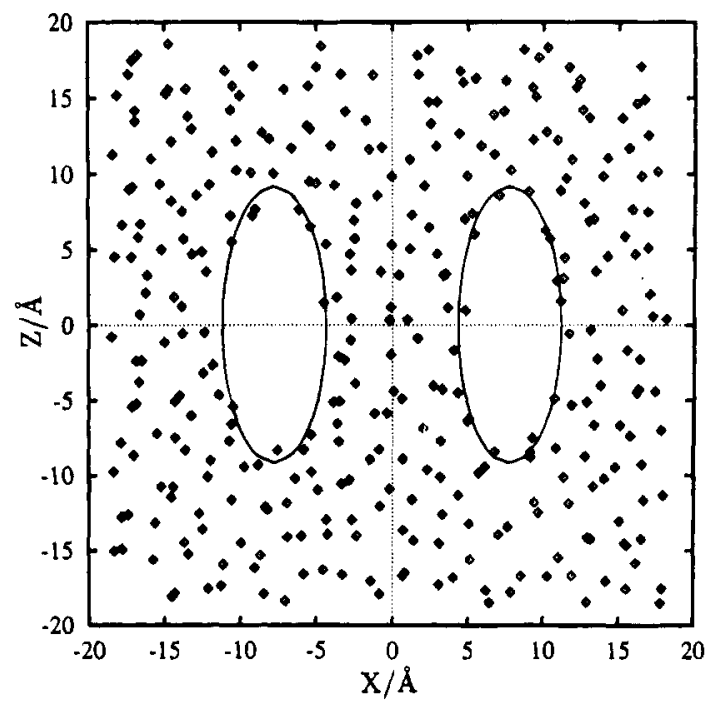

Figure 2. Coordinate projection of the system where the ellipsoidal objects are at a separation $r_{\mathrm{s}}=15.5 \AA$. Obscuring water molecules have been removed, and the ellipsoids have been indicated by the equipotential surface $U_{s w}=k T$. Three intervening water layers are seen.

since it can be independently added to the free-energy curve. For water-water interactions, we have employed the restricted effective representation model of water which uses effective liquid-state charges in a pairwise additive potential, RER(pair). ${ }^{40}$ By using partial charges located at the oxygen and hydrogen sites, the effective pair-potential model is assigned a permanent dipole moment of $2.60 \mathrm{D}$. The monomer geometry of the water molecule is given by a bond length $r_{0}=0.96 \AA$ and a bond angle $\theta_{0}=104.52^{\circ}$. The functional form of the water-water potential is

$$
\begin{aligned}
U_{\mathrm{ww}}\left(\left\{\mathbf{R}_{i}\right\},\left\{\mathbf{R}_{j}\right\}\right)=\frac{C_{12}}{r_{\mathrm{OO}}^{12}}+\frac{C_{6}}{r_{\mathrm{OO}}^{6}}+\frac{C_{4}}{r_{\mathrm{OO}}^{4}}+C_{t} e^{-w_{\mathrm{t}}\left(r_{\mathrm{OO}}-r_{t}\right)^{2}}+ & \sum_{\alpha \in i, \beta \in j}^{\text {atoms }} \frac{q_{\alpha} q_{\beta}}{4 \pi \epsilon_{0} r_{\alpha \beta}}
\end{aligned}
$$

where $\left\{\mathbf{R}_{i}\right\}$ denotes the coordinates of water molecule $i, r_{\mathrm{OO}}$ is 
TABLE 1: RER(pair) Water-Water Pair Potential Parameters ${ }^{a}$

\begin{tabular}{ccccccccc}
\hline$q_{0}, e$ & $q_{\mathrm{H}}, e$ & $\alpha, \AA^{3}$ & $\mathrm{~kJ} /\left(\mathrm{mol} \AA^{12}\right)$ & $\begin{array}{c}C_{6}, \\
\mathrm{~kJ} /\left(\mathrm{mol} \AA^{6}\right)\end{array}$ & $\begin{array}{c}C_{4}, \\
\mathrm{~kJ} /\left(\mathrm{mol} \AA^{4}\right)\end{array}$ & $\begin{array}{c}C_{t}, \\
\mathrm{~kJ} / \mathrm{mol}\end{array}$ & $w_{t}, \AA^{-2}$ & $r_{t}, \AA$ \\
\hline-0.920 & 0.460 & 0.00 & 3500000 & -3100 & 15.0 & -1.000 & 1.5
\end{tabular}

${ }^{a}$ In order to recover the potential energy in $\mathrm{kJ} / \mathrm{mol}$ when using eq $5,1 / 4 \pi \epsilon_{0}$ should be set to 1389.0 . The correction term to be added to the RER(pair) potential due to the self-energy of the dipole moment, $V_{\text {self }}$, is $13.2 \mathrm{~kJ} / \mathrm{mol}$ at $300 \mathrm{~K}$.

the distance between oxygen atoms on water $i$ and $j$, and $r_{\alpha \beta}$ is the radial distance between two atoms on the $i$ th and $j$ th water molecule. The total potential energy was adjusted for the energy cost of creating the effective dipole moments, $V_{\text {self }}{ }^{40,43,44}$ The parameter values for the water model are given in Table 1.

A constant-pressure molecular dynamics algorithm was used in order to maintain the pressure at 1 bar..$^{45}$ The system is thus enclosed in a periodically replicated cubic box with a variable side length of approximately $37 \AA$. The two oblate solute molecules occupy approximately $4 \%$ of the total volume of the simulation cell. The equations of motion were integrated using the Rattle version ${ }^{46}$ of the velocity Verlet algorithm ${ }^{47}$ in order to maintain the internal bond lengths and bond angle constraints of the rigid molecule. A 2.0-fs time step was used. The solutes were kept fixed at their specified separations, $r_{\mathrm{s}}$. Temperatures were maintained at $300 \mathrm{~K}$ by periodically rescaling the velocities. Both the translational and the rotational temperatures were monitored so as to avoid a temperature imbalance between these degrees of freedom. All interactions were spherically truncated at half the box length, which has been shown to be adequate in order to account for the long-range interactions in most water properties. ${ }^{48}$

Thermodynamic and statistical perturbation theory originally due to Zwanzig ${ }^{49,50}$ was used to calculate the relative free energies for difference plate separations. The change in Gibbs free energy between two systems $r_{0}$ and $r_{1}$ containing $N$ particles at a given temperature, $T$, and pressure, $P$, is given by

$$
\Delta G=G\left(r_{1}\right)-G\left(r_{0}\right)=-k T \ln \left\langle e^{-\left[U\left(r_{1}\right)-U\left(r_{0}\right)\right] / k T}\right\rangle_{0}
$$

where $k$ is Boltzmann's constant and the brackets indicates an ensemble average of the exponential of the perturbation in the reference system.

The entropy contribution to the free-energy change in the system can be evaluated from the temperature derivative of the Gibbs free energy to yield ${ }^{51,20}$

$$
-T \delta S=\Delta G-\frac{\left\langle\mathscr{K}_{1} e^{-\Delta V / k T}\right\rangle_{0}}{\left\langle e^{-\Delta V / k T}\right\rangle_{0}}+\left\langle\mathscr{H}_{0}\right\rangle_{0}
$$

where $\mathscr{R}_{1}$ and $\mathscr{H}_{0}$ are the perturbed and the reference Hamiltonians, respectively. Even though this estimator is associated with a large uncertainty as calculated from molecular simulations, it is still useful to gauge the overall entropic response of the system.

A set of simulations with the plate separation $r_{\mathrm{s}}$ between 16.5 and $5.5 \AA$ was carried out in order to characterize the freeenergy change according to eq 6 and eq 7. The distance, $r_{\mathrm{s}}$, was changed in steps of $0.5 \AA$ for the reference states, and the free-energy changes were accumulated in intervals of $\pm 0.10 \AA$ for each reference state. Each reference system was equilibrated for $10.0 \mathrm{ps}$ starting from an independent system. Thermodynamic averages were then calculated from block-averaging five simulations of $5.0 \mathrm{ps}$ each. The error of the free energy for the perturbed systems was estimated as twice the standard deviation obtained from the block averaging procedure,

\begin{tabular}{|c|c|c|c|c|c|}
\hline$r_{\mathrm{s}}, \AA$ & $\begin{array}{c}\Delta G, \\
\mathrm{~kJ} / \mathrm{mol}\end{array}$ & $\begin{array}{l}-T \Delta S \\
\mathrm{~kJ} / \mathrm{mol}\end{array}$ & $r_{\mathrm{s}}, \AA$ & $\begin{array}{c}\Delta G \\
\mathrm{~kJ} / \mathrm{mol}\end{array}$ & $\begin{array}{l}-T \Delta S, \\
\mathrm{~kJ} / \mathrm{mol}\end{array}$ \\
\hline 16.5 & $0.0 \pm 2.5$ & $0 \pm 9$ & 10.5 & $+10.4 \pm 3.4$ & $+50 \pm 12$ \\
\hline 16.0 & -2.8 & $-3 \pm 10$ & 10.0 & & +47 \\
\hline 15.5 & -3 & $-5 \pm$ & 9.5 & & $+40 \pm$ \\
\hline 15.0 & -1.8 & $+6 \pm$ & 9.0 & -8. & $+24 \pm$ \\
\hline 14.5 & +1 & $+18 \pm$ & 8.5 & -20.0 & $+8 \pm$ \\
\hline 14.0 & & $+24 \pm$ & 8.0 & $-32.2 \pm$ & $-10 \pm$ \\
\hline 13.5 & +6 & $+32 \pm$ & 7.5 & $-46.0 \pm$ & $-30 \pm 11$ \\
\hline 13.0 & & $+41 \pm$ & 7.0 & $-58.0 \pm$ & $-46 \pm 10$ \\
\hline 12.5 & & $+41 \pm$ & 6.5 & $-71.0 \pm$ & $-68 \pm 9$ \\
\hline 12 & +5 & $+37 \pm$ & 6.0 & $-86.6 \pm$ & $-93 \pm$ \\
\hline & & $+39 \pm$ & 5.5 & $-100.2 \pm 2.5$ & $-117 \pm 9$ \\
\hline & & & & & \\
\hline
\end{tabular}

TABLE 2: Free-Energy Estimates

$$
\text { error in } X= \pm 2\left[\frac{\sum_{n=1}^{N}\left(X_{i}-X_{\text {ave }}\right)^{2}}{N(N-1)}\right]^{1 / 2}
$$

where $X$ is the estimated property, $X_{\text {ave }}$ is the average, and $N$ is the number of block-averaged systems. As the perturbation technique estimates the change in free energy relative to each reference system simulated, the total free-energy change has to be reconstructed by connecting the individual free-energy curves from each reference system. The error estimate of the freeenergy curve is then propagated forward and backward using the error of the connecting point. ${ }^{51-53}$

The total simulation time was approximately $700 \mathrm{CPU}$ h using an IBM RISC System/6000 Model 580 computer.

\section{Results and Discussion}

3.1. Thermodynamic Properties. The change in free energy (or equivalently the potential of mean force) of the system as a function of plate separation is given in Figure $3 \mathrm{a}$ and Table 2. The maximum separation at $16.5 \AA$ has arbitrarily been assigned a free energy of zero. The free-energy changes can be put on an absolute basis if additional computer experiments are done in order to evaluate the absolute free energy of hydration for the solute, as we see at a separation of $r_{\mathrm{s}}=15.5 \AA$.

It is assumed in the following that for each plate separation, the molecular dynamics run is sufficiently long that equilibrium configurations of water are sampled. Thus, as the plates are moved together "reversibly" from $16.5 \AA$, a first minimum is observed in $\Delta G(r)$ at $15.5 \AA$. From the coordinate projection of Figure 2, it is seen that at this separation, the system supports three intervening water layers. In Figure $3 \mathrm{~b}$, this corresponds to the solvent-induced force being zero. Further reduction in plate separation is associated with a repulsive free-energy change, and at a separation of $12 \AA$, the third water layer is completely expelled, as is evident from Figure 4 . At this separation, there is again a relative minimum in the free energy (the solvent-induced force between the plates is zero because in our model there is no van der Waals force between the plates). We see in Table 3 that the physical properties of the system, such as its average volume and the averaged potential energy 

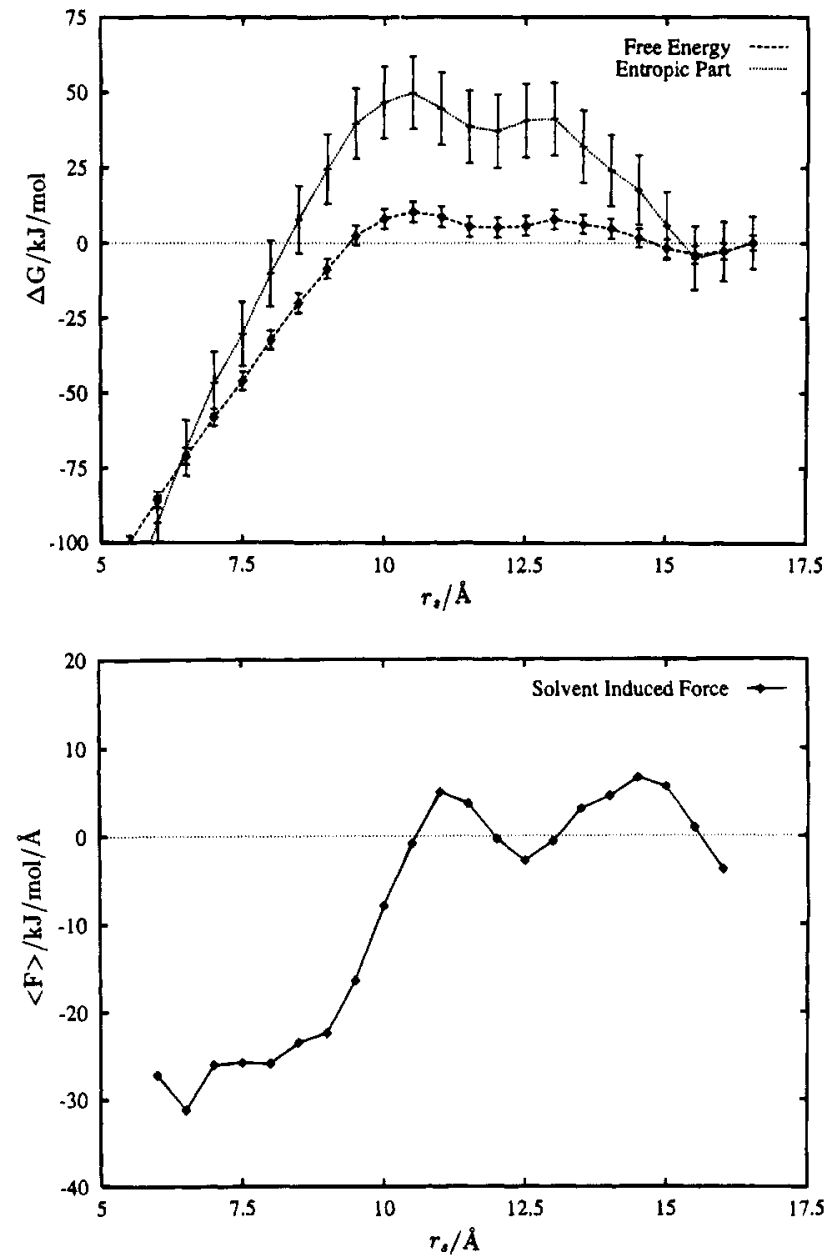

Figure 3. (a, top) Free-energy change of different values for the plate separation, $r_{\mathrm{s}}$, relative to the maximum separation at $r_{\mathrm{s}}=16.5 \AA$. The entropic contribution to the free-energy change. $-T \Delta S$, is also indicated. (b, bottom) Associated solvent-induced force, $\langle F\rangle=\delta \mathrm{G} /$ $\delta \mathrm{r}_{\mathrm{s}}$, derived from a.

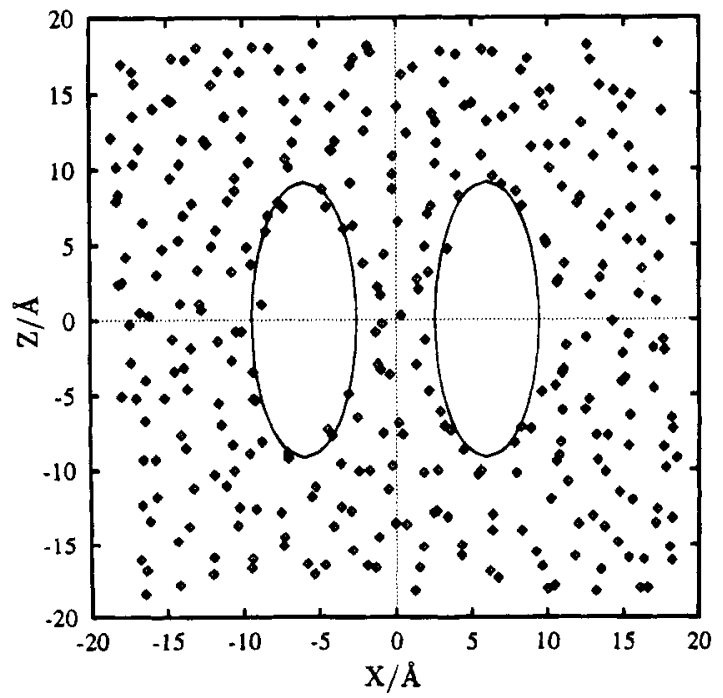

Figure 4. Coordinate projection similar to that of Figure 2 but at a plate separation of $12.0 \AA$. Two interstitial water layers are clearly discernible.

of water-water and water-solute interactions, undergo only small changes at these separations.

The potential of mean force for plates separations $r_{\mathrm{s}} \geq 9.5 \AA$ undulates. Locally stable solvent-separated configurations correspond to the formation of integral numbers of water layers as
TABLE 3: Properties of the Systems at $300 \mathrm{~K}$ and $1 \mathrm{bar}^{b}$

\begin{tabular}{rcccc}
\hline$r_{5}, \AA$ & $\begin{array}{c}\text { vol, } \\
10^{3} \AA^{3}\end{array}$ & $\begin{array}{c}U_{\mathrm{sw}}, 10^{2} \\
\mathrm{~kJ} / \mathrm{mol}\end{array}$ & $\begin{array}{c}\left(U_{\mathrm{ww}}-V_{\text {self }}\right){ }^{a} \\
\mathrm{~kJ} / \mathrm{mol} \mathrm{of} \mathrm{H}_{2} \mathrm{O}\end{array}$ & $\begin{array}{c}\text { total } \\
\mathrm{nn}\end{array}$ \\
\hline 16.5 & 51.0 & 1.14 & -41.0 & 51 \\
16.0 & 51.0 & 1.17 & -41.0 & 49 \\
15.5 & 50.9 & 1.21 & -41.0 & 52 \\
15.0 & 50.9 & 1.25 & -40.9 & 55 \\
14.5 & 50.8 & 1.19 & -41.1 & 53 \\
14.0 & 50.9 & 1.15 & -41.1 & 51 \\
13.5 & 51.0 & 1.16 & -41.0 & 53 \\
13.0 & 50.9 & 1.19 & -41.0 & 54 \\
12.5 & 51.0 & 1.15 & -41.0 & 51 \\
12.0 & 51.0 & 1.13 & -41.0 & 52 \\
11.5 & 50.9 & 1.12 & -41.1 & 52 \\
11.0 & 50.9 & 1.21 & -41.0 & 53 \\
10.5 & 50.9 & 1.20 & -41.0 & 54 \\
10.0 & 51.0 & 1.13 & -41.0 & 49 \\
9.5 & 51.5 & 0.96 & -41.1 & 39 \\
9.0 & 51.5 & 0.90 & -41.0 & 40 \\
8.5 & 51.5 & 0.89 & -40.9 & 38 \\
8.0 & 51.6 & 0.77 & -41.1 & 33 \\
7.5 & 51.5 & 0.76 & -41.0 & 36 \\
7.0 & 51.4 & 0.81 & -41.1 & 39 \\
6.5 & 51.4 & 0.76 & -41.0 & 33 \\
6.0 & 51.3 & 0.78 & -41.1 & 34 \\
5.5 & 51.2 & 0.78 & -41.1 & 35
\end{tabular}

${ }^{a}$ The potential energy of the neat liquid is $-41.6 \mathrm{~kJ} / \mathrm{mol}^{40}{ }^{\circ} \Delta U\left(r_{\mathrm{s}}\right)$ is defined as $\left(U_{\mathrm{tot}}\left(r_{\mathrm{s}}\right)-U_{\mathrm{tot}}(16.5 \AA)\right)$ where the total potential energy of the system is given by $U_{\text {tot }}=U_{\mathrm{sw}}+U_{\mathrm{ww}} N_{\mathrm{w}}$ with $N_{\mathrm{w}}=1656$ for the simulated system. The error in the given quantities is as follows: volume $\pm 0.05 \times 10^{3} \AA^{3} ; U_{\text {sw }}, \pm 0.03 \times 10^{2} \mathrm{~kJ} / \mathrm{mol} ; U_{\mathrm{ww}}-V_{\text {self, }} \pm 0.05$ $\mathrm{kJ} / \mathrm{mol}$; total $\mathrm{nn}, \pm 2$ molecules.

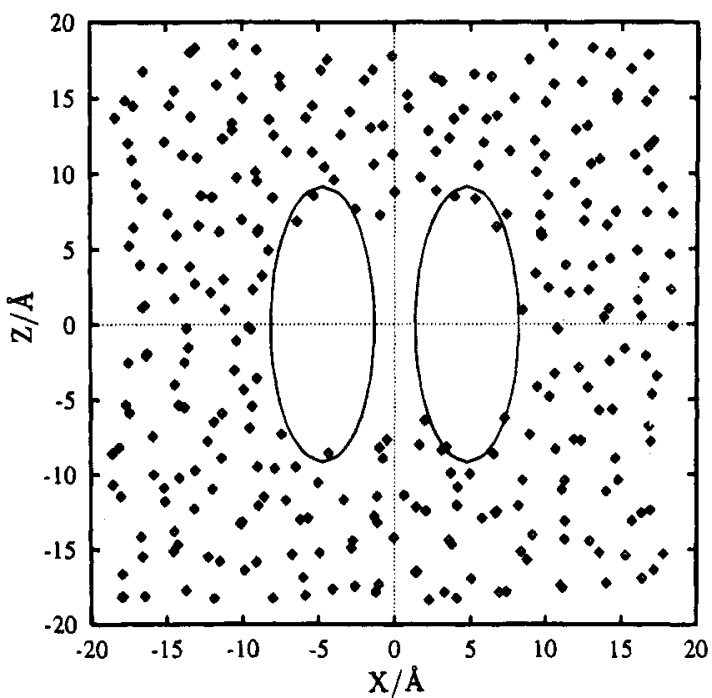

Figure 5. Coordinate projection similar to that of Figure 2 but at a plate separation of $9.5 \AA$. All interstitial water molecules have now been expelled from the space between the plates.

can be seen from Figures 2, 4, and 5. This is superficially similar to the behavior of small apolar spherical solutes where clathrate-like water cages have been thought to be responsible for the stabilization of solvent-separated pairs. Thus, for sufficiently large plates, where clathrate cages cannot form, water layering provides a means of accommodating large flat plates at specific distances which are integral number of the diameter of a water molecule. Further investigation of the longrange propagation of these water layers is hampered by the need to study much larger systems.

The total number of water molecules in the first solvent shell around the plates is also given in Table 3. A water molecule is said to be in the first shell if it is within $0.665 \AA$ from the equipotential surface given in Figure 1 . As the plates are 
brought together, this number doesn't change much until a separation of $9.5 \AA$ is reached.

The plates when reversibly moved together to a separation of $9.5 \AA$ expel the remaining interstitial water molecules. It should be noted that the plates do not start overlapping until they are closer than $r_{\mathrm{s}}=\sigma_{\|}=3.1 \AA$. Although a single water layer can fit between the ellipsoids at the separation of $9.5 \AA$, a single water layer is not seen. Water molecules are instead displaced from the slightly curved surface to the sides of the ellipsoid and into the surrounding bulk fluid. In Figure 5, a typical corresponding coordinate projection is given which shows the empty hourglass-shaped void formed between the plates. This dewetting transition probably occurs because hydrogen bonding stabilizes the water layers between the plates, and when only a single water layer can fit between the plates, one loses sufficient hydrogen-bond energy to make it more favorable for these water molecules to move into the bulk. It is very likely that the dewetting transition is also entropically driven. From Table 3, we can see that the response of the system to the formation of the empty cavity is to increase the total volume of the system (at constant pressure). The change in volume corresponds to about 20 water molecules at $1.0 \mathrm{~g} / \mathrm{cm}^{3}$, which is roughly the number of water molecules that would have been needed to form a single water layer between the plates if one formed. Thus, the total volume accessible to the water molecules remains essentially unchanged. With the removal of the interstitial waters, the solute-water energies decrease due to the smaller number of nearest-neighbor waters around the plates. The plates are now less exposed to the surrounding fluid. If the plates were brought to a separation of $r_{\mathrm{s}}=0$, so that they overlap, the solute-water energies would be reduced by half compared to the solute-water energies at $r_{\mathrm{s}}>15 \AA$, where two fully developed hydration shells are present.

When the plates are brought close enough for the dewetting transition (the ejection of the water molecules in the interstitial region), there is a sharp decrease in $\Delta G(r)$. Associated with this is a reduction in the number of water molecules in contact with the plates and a concomitant decrease in the solute-water interaction energy and the solute-water surface area. Once the dewetting transition occurs with the formation of an interstitial void region, the volume and surface area of the void region is a continuously decreasing function of the plate separation (for distances shorter than $r_{\mathrm{s}}<9.5 \AA$ ); in this regime, the area of contact between the plates and water remains constant. The change in free energy is then approximately linear in the plate separation and amounts to an apparent constant attractive force of $25(\mathrm{~kJ} / \mathrm{mol}) / \AA$ between the solute plates. This force is solely due to the presence of the solvent.

The void space between the plates decreases in volume as $r_{s}$ decreases. This volume change can be approximated by a cylindrical volume change by $\Delta \mathscr{T}^{\prime}=\sigma_{\perp}{ }^{2} \Delta r_{\mathrm{s}}$. Similarly, the change in exposed surface area can be approximated by $\Delta \mathcal{A}=$ $2 \pi \sigma_{\perp} \Delta r_{\mathrm{s}}$. Both of these quantities are linear in the change of plate separation. The decrease of the exposed surface area is energetically favorable. In fact, an estimate of the surface tension between the liquid-vapor interface of water can be made from

$$
\gamma_{1 \mathrm{v}}=\frac{\Delta G}{\Delta \mathscr{A}}
$$

This gives an estimate of $\gamma_{1 v}$ to be $70 \mathrm{dyn} / \mathrm{cm}(0.44(\mathrm{~kJ} / \mathrm{mol}) /$ $\AA^{2}$ ), similar but not in agreement with the independently measured value of $100 \mathrm{dyn} / \mathrm{cm}$ for a free planar surface of this water model. ${ }^{37}$ Since the surface area of the hourglass-shaped interface is smaller than the cylindrical estimate, use of the

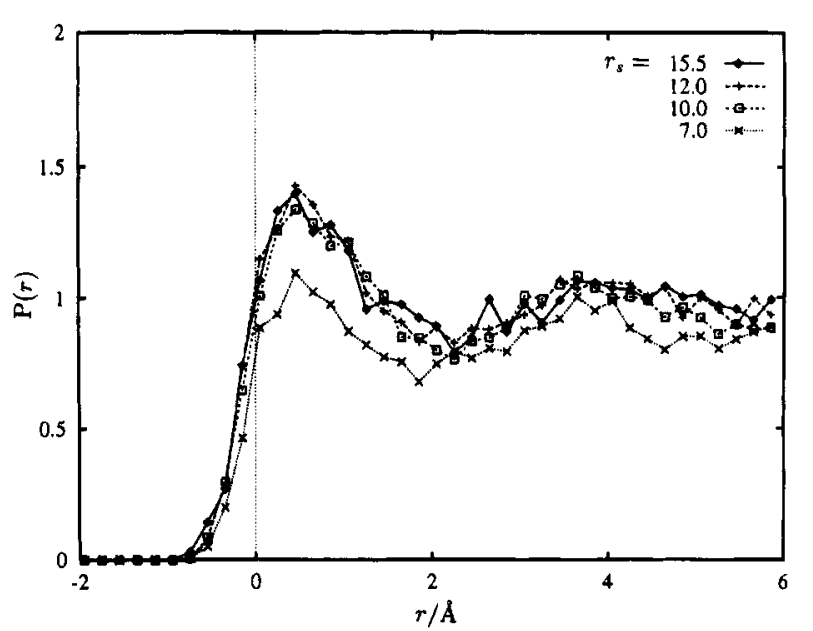

Figure 6. Distribution of water molecules along the surface normal of the ellipsoids. The equipotential surface $U_{\mathrm{sw}}=k T$ is located at $r=$ 0.0 in the figure.

correct surface area will give rise to a larger value of $\gamma_{\mathrm{lv}}-\mathrm{a}$ value closer to $100 \mathrm{dyn} / \mathrm{cm}$. It can be argued that if the plate is weakly hydrophobic, as in our case, the volume denied the waters resembles a vacuous bubble and the free-energy cost of maintaining the water-solute interface should closely resemble that of the water liquid-vapor interface..$^{54,55}$ Of course, this argument ignores the possibility that water orients differently at planar than at nonplanar interfaces ${ }^{32}$ and there should then be a curvature dependence of the surface tension. Since the physical properties of the surface should depend on the shape of the surface, it is virtually impossible to give an a priori determination of the "surface tension"-like coefficient $\gamma_{1 \mathrm{v}}$ in eq 9.

3.2. Hydrophobic Hydration. The distribution of water molecules in annular ellipsoidal shells around the oblate solute molecule was investigated by determining the number of water molecules, $\Delta N$, in a shell of annular volume

$$
\Delta v=\frac{4 \pi}{3} \frac{\sigma_{\|}}{\sigma_{\perp}} r^{2} \Delta r
$$

where $r$ is the distance from the center of the oblate solute to the beginning of the shell along a direction parallel to one of the $\sigma_{\perp}$ axes and $\Delta r$ is the thickness of the shell. In essence, each shell is labeled by $r$, the distance of closest approach of its inner surface to the center of the solvent-excluded volume of the ellipsoidal solute. We then define the relative density of molecules in the shell,

$$
P(r) \equiv \frac{\varrho(r)}{\varrho}=\frac{\Delta N}{\varrho \Delta v}
$$

where $Q$ is the bulk density. $P(r)$ gives the relative water density in a shell located at distance $r$ from the ellipsoidal surface. For a spherical solute, $\sigma_{\perp}=\sigma_{\|}, P(r)$ reduces to the ordinary radial distribution function $g^{(2)}(r)$.

In Figure 6, $P(r)$ is plotted for three separations $\left(r_{\mathrm{s}}=15.5\right.$, $12.0,10.0 \AA)$ of the stacked plates with intervening waters surrounding the solutes and one $\left(r_{\mathrm{s}}=7.0 \AA\right)$ in which there are none. Water is able to closely approach and even penetrate slightly into the equipotential ( $\left.U_{\text {sw }}=k T\right)$ surface, in accord with the weakly repulsive interaction potential. No long-range structural ordering is observed, and the distribution functions approach their bulk value within $2 \AA$ of the surface. The distribution of solvent molecules around the plates is similar for all separations where the solutes are completely surrounded by water molecules. There is no noticeable difference between 
$P(r)$ 's at either free-energy minima or free-energy maxima. Naturally, the distribution of waters is reduced at shorter interplate distances when there are no intervening water molecules between the plates, as for $r_{\mathrm{s}}=7.0 \AA$. The orientational distribution of shell waters was also investigated with the same result that shell waters have virtually the same orientational ordering at the solute interfaces regardless of the plate-plate separation. This ordering is short ranged, and the bulk orientation, a random orientation with respect to the surface, is established immediately outside the first shell.

\section{Conclusion}

In this study, we have determined the potential of mean force $\Delta G(r)$ as a function of plate separation between two large parallel oblate ellipsoids, or platelike molecules, in water at constant pressure. The volume of each ellipsoid displaces as many as 40 water molecules. Each plate interacts repulsively with each water molecule through a short-ranged potential specified by eq 1 . In effect, this potential disturbs the local ordering of neighboring water molecules. The direct solvent reorganization due to this cavity is found to be weak and does not extend out to more than one solvation shell. As shown in Figure $3 \mathrm{a}$ and Figures 2, 4, and 5, the relative minima in the potential of mean force correspond to two or three water layers between the plates. $\Delta G(r)$ decreases monotonically as the plates are brought closer than $9.5 \AA$. This is the distance at which there is a dewetting transition (see Figure 5) in which all interstitial water molecules are expelled; that is, one never observes a monolayer of water between the plates. In this dewetting regime, it is found that (a) the plates are separated by a vacuous hole; (b) $\Delta G(r)$ is a linear function of the plate separation; (c) the interfacial area between the vacuum and water is, to a very good approximation, a linear function of the plate separation; and (d) the potential of mean force, $\Delta G(r)$, is a linear function of $\Delta A$, the area of the interface between the vacuum and liquid water,

$$
\Delta G=\gamma_{1 v} \Delta A
$$

where $\gamma_{\mathrm{lv}}$ is a constant of proportionality. In cgs units $\gamma_{\mathrm{lv}}$ is found to be $70 \mathrm{dyn} / \mathrm{cm}$, which corresponds to a free energy per $\AA^{2}$ of $0.44 \mathrm{~kJ} / \mathrm{mol}$. It is important to note, however, that the water-vacuum interfacial area was estimated by approximating the void region by a cylinder. In truth, it has an hourglass shape and its surface area is smaller than that of the approximating cylinder. Thus, the value of $\gamma_{1 v}$ reported is larger than would be determined if an accurate value of the surface area were used and it would be closer to the liquid-vapor surface tension which we have determined to be $\gamma=100 \mathrm{dyn} / \mathrm{cm}$ for the RER model of water. ${ }^{37}$ The plates are driven together by the solvent's need to minimize the solvent-exposed surface area, but this happens only locally. Thus, the hydrophobic driving force appears to arise from the need for the system to minimize the surface free energy.

It is important to recognize that fixed-charge pair potential models of water are not expected to have surface tensions in agreement with experiment because these models do not include many-body interactions. The gas-liquid surface tension for the RER model has been determined as function of temperature. ${ }^{37}$ At $300 \mathrm{~K}$, we find that $\gamma=100 \mathrm{dyn} / \mathrm{cm}$. By contrast, we have determined that the SPC/E model ${ }^{43}$ has $\gamma=55 \mathrm{dyn} / \mathrm{cm}$, in close agreement with the reported value of $59 \mathrm{dyn} / \mathrm{cm}^{56,57}$ of the TIP4P model. ${ }^{26}$ None of these surface tension calculations include Axilrod-Teller three-body dispersion interactions. ${ }^{58}$ In inert fluids like $\mathrm{Ar}$, it is known that these three-body interactions have a major effect on the surface tension. For example, Ar at its triple point has a measured surface tension of $\gamma=13 \mathrm{dyn} /$ $\mathrm{cm}$, whereas simulations based only on an untruncated pair potential yield $\gamma=18 \mathrm{dyn} / \mathrm{cm} .{ }^{59}$ When the Axilrod-Teller terms are added to the two-body interactions, simulations yield $\gamma=13 \mathrm{dyn} / \mathrm{cm},{ }^{60}$ in agreement with experiment. Thus, threebody terms reduce the surface tension from what pair interactions predict (back of the envelope estimates for water give a reduction of $\sim 10 \mathrm{dyn} / \mathrm{cm}$ ) and if introduced into the calculation of the surface tension of water using the RER potential should reduce it from $100 \mathrm{dyn} / \mathrm{cm}$ and thus bring it closer to the experimental value for the air-water interface $(\gamma=72 \mathrm{dyn} /$ $\mathrm{cm})$. Nevertheless, our discussion of interfacial tensions and the lack of agreement between the two coefficients reported in the preceding paragraph is of theoretical interest only.

Because water molecules next to the interstitial vacuum should behave similarly to water molecules next to the gasliquid interface, it could be expected that it would be the gasliquid surface tension that describes the process. ${ }^{55}$ However, because this interface is not flat, we expect that curvature corrections predicted by Tolman, ${ }^{61,62}$ and shown by us to be important for small cavities, ${ }^{32}$ would lead to an effective "surface tension" that is different from the gas-liquid surface tension. These differences are ascribed to the detailed nature of water conformations at different surfaces. There appears to be no easy way of correlating these effective surface tension coefficients with the macroscopic surface tension.

The structure of water does not appear to be strongly perturbed by the excluded volume of our hydrophobic solutes. There are no strong structural or orientational perturbations compared to correlations between water molecules in the neat liquid. Bulk properties are reached within one water layer. Even though the perturbation of the water structure per se is small, each water molecule at the surface is penalized both energetically (lack of hydrogen bonds) and entropically (the locally available volume is reduced). It is the reduction of the watersolute surface at the dewetting transition that contributes to the large negative free energy $(-T \Delta S)$ immediately below $10 \AA$.

It is important to note that all of the above conclusions would be different if the simulations were instead carried out in a constant volume ensemble. Then the 20 water molecules ejected into the bulk solvent when the 2 ellipsoids are brought to $10 \AA$ in the constant-pressure ensemble would not be ejected in the constant-volume ensemble and the potential of mean force would probably exhibit a repulsive behavior. Of course, if the simulation volume were made large enough, the two ensembles would give essentially the same results, but this would require unfeasibly large volumes for practical simulations. Fortunately, real experiments are carried out at constant pressure not constant volume. For small molecular solutes, the differences between isochoric and isobaric simulations will be negligible.

We believe that the observed behavior here will be robust with respect to different water potentials.

Acknowledgment. This work was supported by a grant from the National Institutes of Health (GM43340-01A1) and was done at the NIH Biotechnology Resource Center at Columbia University. We acknowledge that the simulations were carried out on a network of IBM RISC/6000 workstations purchased on an NSF department instrument grant. We thank Prof. Barry Honig for useful discussions.

\section{References and Notes}

(1) Tanford, C. The Hydrophobic Effect; Wiley: New York, 1973. 1974.

(2) Ben-Naim, A. Water and Aqueous Solutions; Plenum: New York, 
(3) Franks, F. In Water; A Comprehensive Treatise; Franks, F., Ed.; Plenum: New York, 1975; Vol. 4.

(4) Chan, D. Y. C.; Mitchell, D. J.; Ninham, B. W.; Pailthorpe, B. A. In Water; A Comprehensive Treatise; Franks, F., Ed.; Plenum: New York, 1979; Vol. 6.

(5) Blokzijl, W.; Engberts, J. B. F. N. Angew. Chem., Int. Ed. Engl. 1993, 32, 1545-1579.

(6) Pratt, L. R.; Chandler, D. J. Chem. Phys. 1977, 67, 3683-3704.

(7) Pratt, L. R.; Chandler, D. J. Chem. Phys. 1980, 73, 3434-3441. 2981 .

(8) Pangali, C.; Rao, M.; Berne, B. J. J. Chem. Phys. 1979, 71, 2975-

(9) Pangali, C.; Rao, M.; Berne, B. J. J. Chem. Phys. 1979, 71, 29822990

(10) Tani, A. Mol. Phys. 1984, 51, 161-173.

(11) Pratt, L. R. In Annual Review of Physical Chemistry; Rabinovitch, B. S., Schurr, J. M., Strauss, H. L., Eds.; Annual Reviews: New York, 1985; Vol. 36.

(12) Pratt, L. R.; Chandler, D. Methods Enzymol. 1986, 127, 48-63. 880 .

(14) Ravishanker, G.; Mezei, M.; Beveridge, D. L. Faraday Symp. Chem. Soc. 1982, 17, 79-91.

(15) Watanabe, K.; Andersen, H. C. J. Phys. Chem. 1986, 90, 795802.

(16) Tobias, D. J.; Brooks, III, C. L. Chem. Phys. Lett. 1987, 142, 472476.

(17) Jorgensen, W. L.; Buckner, J. K.; Boudon, S.; Tirado-Rives, J. J. Chem. Phys. 1988, 89, 3742-3746.

(18) Guillot, B.; Guissani, Y.; Bratos, S. J. Chem. Phys. 1991, 95, 36433648.

(19) Smith, D. E.; Zhang, L.; Haymet, A. D. J. J. Am. Chem. Soc, 1992, $114,5875-5876$ 6454 . 652.

(21) van Belle, D.; Wodak, S. J. J. Am. Chem. Soc. 1993, 115, 647-

(22) Dang, L. X. J. Chem. Phys. 1994, 100, 9032-9034.

(23) Wallqvist, A.; Berne, B. J. Chem. Phys. Lett. 1988, 145, 26-32.

(24) Berne, B. J.; Wallqvist, A. Chem. Scr. 1989, 29A, 85-91.

(25) Stillinger, F. H.; Rahman, A. J. Chem. Phys. 1972, 57, 12811292.

(26) Jorgensen, W. L. J. Chem. Phys. 1982, 77, 4156-4163.

(27) Berendsen, H. J. C.; Postma, J. P. M.; van Gunsteren, W. F Hermans, J. In Intermolecular Forces; Pullman, B., Ed.; D. Reidel: Dordrecht, 1981; pp 331-342.

(28) Lee, C. Y.; McCammon, J. A.; Rossky, P. J. J. Chem. Phys. 1984 $80,4448-4455$.

(29) Zichi, D. A.; Rossky, P. J. J. Chem. Phys. 1985, 83, 797-808.

(30) Zichi, D. A.; Rossky, P. J. J. Chem. Phys. 1986, 84, 2814-2822.
(31) Wallquist, A. Chem. Phys. Lett. 1990, 165, 437-442.

(32) Wallqvist, A.; Berne, B. J. J. Phys. Chem., this issue.

(33) Israelachvili, J.; Pashley, R. Nature 1982, 300, 341-342.

(34) Israelachvili, J. N.; Pashley, R. M. Nature 1983, 306, 249-250.

(35) Pashley, R. M.; McGuiggan, P. M.; Ninham, B. W.; Evans, D. F. Science 1985, 229, 1088-1089.

(36) Tsao, Y.-H.; Evans, D. F.; Wennerstrom, H. Science 1993, 262, $547-550$.

(37) Wallqvist, A.; Berne, B. J. Liquid-vapor interface of polarizable and nonpolarizable water. Manuscript in preparation, 1994.

(38) Richards, F. M. Annu. Rev. Biophys. Bioeng. 1977, 6, 151

(39) Pratt, L. R.; Pohorille, A. Proc. Natl. Acad. Sci. U.SA. 1992, 89, 2993.

(40) Wallqvist, A.; Berne, B. J. J. Phys. Chem. 1993, 97, 13841-13851

(41) Gay, J. G.; Berne, B. J. J. Chem. Phys. 1981, 74, 3316-3319.

(42) Berne, B. J.; Pechukas, P. J. Chem. Phys. 1972, 56, 4213-4216.

(43) Berendsen, H. J. C.; Grigera, J. R.; Straatsma, T. P. J. Phys. Chem. 1987, 91, 6269-6271.

(44) Watanabe, K.; Klein, M. L. Chem. Phys. 1989, 131, 157-167.

(45) Berendsen, H. J. C.; Postma, J. P. M.; van Gunsteren, W. F.; DiNola, A.; Haak, J. R. J. Chem. Phys. 1984, 81, 3684-3690.

(46) Andersen, H. C. J. Comput. Phys, 1983, 52, 24-34.

(47) Swope, W. C.; Andersen, H. C.; Berens, P. H.; Wilson, K. R. J. Chem. Phys. 1982, 76, 637-649.

(48) Wallqvist, A.; Teleman, O. Mol. Phys. 1991, 74, 515-533.

(49) Zwanzig, R. W. J. Chem. Phys. 1954, 22, 1420.

(50) Beveridge, D. L.; DiCapua, F. M. Annu. Rev. Biophys. Biophys. Chem. 1989, 18, 431-492.

(51) Postma, J. P. M.; Berendsen, H. J. C.; Haak, J. R. Faraday Symp. Chem. Soc. 1982, 17, 55-67.

(52) Linse, P. J. Am. Chem. Soc. 1992, 114, 4366-4373.

(53) Linse, P. J. Am. Chem. Soc. 1993, 115, 8793-8797.

(54) Widom, B. J. Chem. Phys. 1965, 43, 3829.

(55) Stillinger, F. H. J. Soln. Chem., 1973, 2, 141-158.

(56) Wilson, M. A.; Pohorille, A.; Pratt, L. R. J. Phys. Chem. 1987, 91 , $4873-4878$

(57) Wilson, M. A.; Pohorille, A.; Pratt, L. R. Hydrophobic effects from cavity statistics. In Proceedings of the EBSA (Association of European Biophysical Societies) 1992 International Workshop in Water-Biomolecule Interactions; Palma, M. U., Palma-Vittorelli, M. B., Parak, F., Eds.; Societa Italiana di Fisica: Rome, 1992.

(58) Axilrod, B. M.; Teller, E. J. Chem. Phys. 1943, 11, 299-300

(59) Miyazaki, J.; Barker, J. A.; Pound, G. M. J. Chem. Phys. 1976, $64,3364-3369$

(60) Barker, J. A.; Klein, M. L. Phys. Rev. 1973, B7, 4707.

(61) Tolman, R. C. J. Chem. Phys. 1949, 17, 333.

(62) Buff, F. P. J. Chem. Phys. 1951, 19, 1591.

JP942646H 